\title{
Exploring Fraunhofer diffraction through Tracker and spreadsheet: An alternative lab activity for distance learning
}

\author{
E. Pratidhina ${ }^{a, b}$, W. Sunu Brams Dwandaru ${ }^{a}$, and H. Kuswanto ${ }^{a}$ \\ ${ }^{a}$ Physics Education, Postgraduate Program, Universitas Negeri Yogyakarta, Jl. Colombo No.1, \\ Karang Malang, Caturtunggal, Depok, Sleman, Daerah Istimewa Yogyakarta 55281. \\ ${ }^{b}$ Department of Physics Education, \\ Widya Mandala Catholic University Surabaya, Jl. Kalijudan 37 Surabaya 60114.
}

Received 30 May 2020; accepted 17 June 2020

In this paper, we present an alternative for physics laboratory activity related to Fraunhofer diffraction in distance learning. The activity utilizes a demonstration video from MIT Open CourseWare, Tracker software, and spreadsheet. An online demonstration video is used because it is the most accessible resource during undesirable conditions such as COVID 19 pandemic. In the activity, students can explore diffractions phenomena with multiple slits. The effect of slit spacing and slit numbers to the intensity of light is investigated trough spectral analysis with Tracker. The investigation is followed by a discussion through the mathematical approach and visualization with spreadsheets. It will enrich students with a theoretical explanation of the observation. This distance learning activity allows students to develop their science process skills, mathematical and computational thinking skills, and conceptual understanding of Fraunhofer diffraction.

Keywords: Fraunhofer diffraction; Tracker; spreadsheet; distance learning.

PACS: 01.40.-d

\section{Introduction}

Recently, distance learning and blended learning are popular. Notably, during the undesirable pandemic, the governments, school principals, and teachers are forced to change traditional classrooms into the digital classes. In a short time, teachers have to think about how to teach their students effectively in distance learning mode. One efficient way is to use existing resources and technologies.

For physics teachers, the challenge is migrating lab activities to the digital model. Several experiment resources are available online; one of them is Demonstrations in physical optics provided by MIT Open Course Ware [1]. The resources include various demonstrations about optical phenomena such as polarization, diffraction, and propagation in optical fiber. That kind of resource can be used to teach physics in distance learning mode. Instead of taking data by hands-on measurement, students can take data by video analysis using software such as Tracker.

Tracker is useful and free software in physics education, which is developed on the Open Source Physics Java code library. People use it for video analysis and physics modeling. By using Tracker, users can track an object's position, velocity, and accelerations [2]. It also has a calibration feature to calibrate the length quantity in the video to the real one. Moreover, the RGB line profile feature in Tracker can be used to analyze spectra.

Tracker has been used for pedagogical purposes and complex physics experiments. Educators and scientist use Tracker to teach and study various topics such as vector object falling in liquid and air [3], projectile motion [4,5], mechanical energy [6], kater pendulum [7], damped harmonic oscillation [8], torsion [9], impulse [10], astronomy [11], and
DOI: https://doi.org/10.31349/RevMexFisE.17.285

optical geometry [12]. Tracker is a potential tool to promote and guide the student as a scientist. Students can explore the laws of nature with Tracker. By using video modeling, students can practice to ask questions, use models, and develop mathematical and computational thinking [13]. It also encourages students to think creatively and improve their physics learning performance [14].

Spreadsheets such as Ms. Excel are powerful computing and graphical tools. In physics education, we can build simple simulations and visualization in the spreadsheet when we are discussing a physics equation $[15,16]$.

In this work, we present the use of these two didactic tools, i.e., Tracker and spreadsheet, for a distance learning activity in physics. We utilize Tracker to analyze the Fraunhofer diffraction pattern at double and multiple slits. Instead of record a video from a direct experiment, we use the available demonstration video resources from MIT Open Course Ware. Then, the observed pattern from Tracker analysis is compared to the theory by utilizing the spreadsheet program, Ms. Excel.

\section{Theory of diffraction}

Phenomena that can prove the wave nature of light is diffraction. It occurs when a wavefront portion is obstructed in some way or encounters one (or many) slits, and the wavefront is altered in amplitude or phase. Segments of wavefront which propagate beyond the obstacle interfere and construct diffraction pattern [17]. There are two kinds of diffractions, i.e., Fresnel diffraction and Fraunhofer diffractions. In Fraunhofer diffraction, the incoming and outgoing waves approach must be planar over the extent of the obstacles. Fraunhofer diffraction occurs when the distance between the obstacle and 
the screen is far enough.

The obstacles for Fraunhofer diffraction can be a single slit, double slits, and multiple slits. The multiple slits can be a diffraction grating. For Fraunhofer diffraction at $N$ parallel slits, the intensity distribution is given by Eq. (1).

$$
I(\theta)=I_{0}\left(\frac{\sin \beta}{\beta}\right)^{2}\left(\frac{\sin N \alpha}{\sin \alpha}\right)^{2},
$$

with,

$$
\begin{aligned}
\alpha & \equiv \frac{\pi(b+d) \sin \theta}{\lambda}, \\
\beta & \equiv \frac{\pi d}{\lambda} \sin \theta,
\end{aligned}
$$

where $d$ is the slit width, $b$ is the slit spacing, $\lambda$ is the wavelength of the light, $\theta$ is the observation angle, and $I_{0}$ is the intensity of the center maxima, i.e., the intensity in the $\theta=0$ rad.

In Fraunhofer diffraction with multiple slits, there are regularly spaced principal maxima, and some smaller maxima which lay in between the principal maxima. The principal maxima spacing on the screen follows relation:

$$
\sin \theta_{n}=\frac{n \lambda}{S_{n}}, \quad n=0, \pm 1, \pm 2, \ldots
$$

By using approximation $\sin \theta_{n} \approx(\Delta / L)$, Eq. (2) can be written as:

$$
S_{n}=\frac{\operatorname{Ln} \lambda}{\Delta}
$$

where $S_{n}$ is the fringe spacing on the screen, $L$ is the distance between the slit and the screen, $\lambda$ is the wavelength, $\Delta$ is the slit separation, and $n$ is an index of the maxima. For diffraction grating, slit separation can be calculated from $\Delta=(1 /$ grating constant $)$.

\section{Method}

We use the Fraunhofer diffraction demonstration video available in MIT Open Couse Ware [1]. The diffraction pattern in the video is analyzed by using Tracker software. Tracker software allows tracking line profiles, which give the data about intensity (luma) at specific points. Tracker also allows calibration to match the dimension, such as length, in the video to the real dimension. Figure 1 shows how the setup of Tracker looks like.

When we start video analysis in Tracker, we have to import the video by selecting File $>$ Import $>$ Video; then we choose the video that we want to analyze. For calibration, we need to select Track Menu $>$ New $>$ Calibration Tool $>$ Calibration Stick. We can mark two calibration points by using a shifting keyboard and click. After two points are marked, we have to input the actual distance of those two points. By

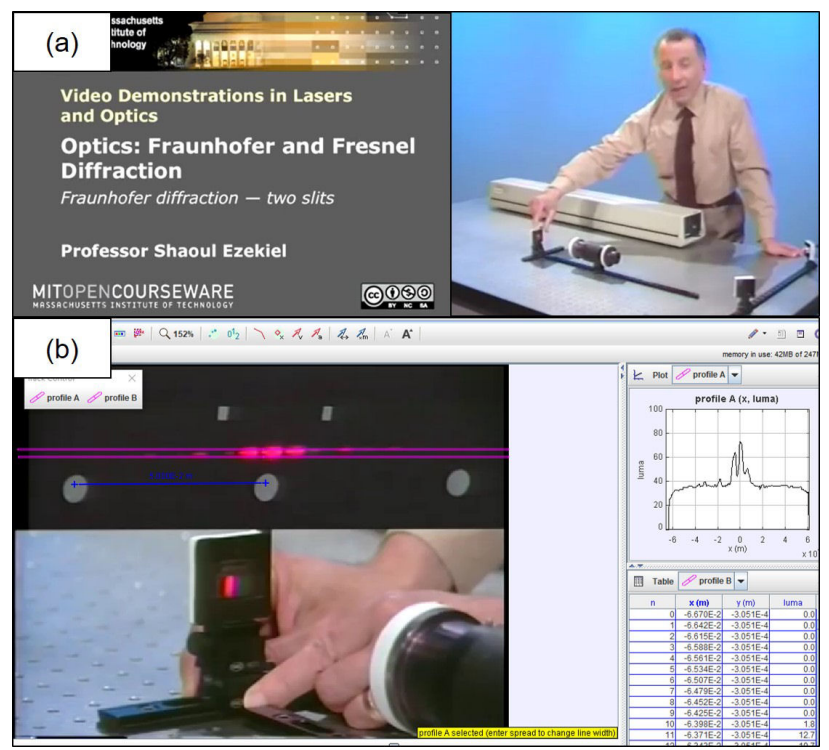

FIGURE 1. (a) Fraunhofer diffraction experiment presented on MIT Open Courseware video demonstration. (b) Diffraction pattern analysis using Video Tracker Software.

doing this, the length of objects in the video has been calibrated to their actual length. For intensity analysis, we need to choose Track Menu $>$ New $>$ Line Profile. We can select the region which we want to analyze by pressing shift keyboard and mouse click. The line region can be expanded by mouse click and drag. The intensity and position data will be presented as a graph and table on the right side of the Tracker program.

After we get the data about point position and intensity in Tracker, we bring them to a spreadsheet program to plot the spectra. Then, we plot Eq. (1) with the given parameters in the experiment to provide a theoretical comparison. In this work, we conducted 3 investigations, i.e.

a. Double Slits Experiment with Slit Spacing Variation, in which we use a video entitled "Fraunhofer Diffraction-Two Slits” in MIT Open Couse Ware [18].

b. Multiple Slits Experiment with Number of Slit Variation, in which we use the first part of a video entitled "Fraunhofer Diffraction-Multiple Slits" in MIT Open Couse Ware [19].

c. Diffraction Grating Experiment with Variation of Grating Constant, in which we use the second part of a video entitled "Fraunhofer Diffraction-Multiple Slits" in MIT Open Couse Ware [19].

\section{Results}

\subsection{Double slits experiment with slit spacing variation}

In the experiment with double slits, the slit spacing is adjusted then the diffraction spectra are analyzed. The slit spacing is varied to be 150,200 , and $300 \mu \mathrm{m}$. The diffraction pattern obtained from analysis with Tracker is depicted in Fig. 2. 


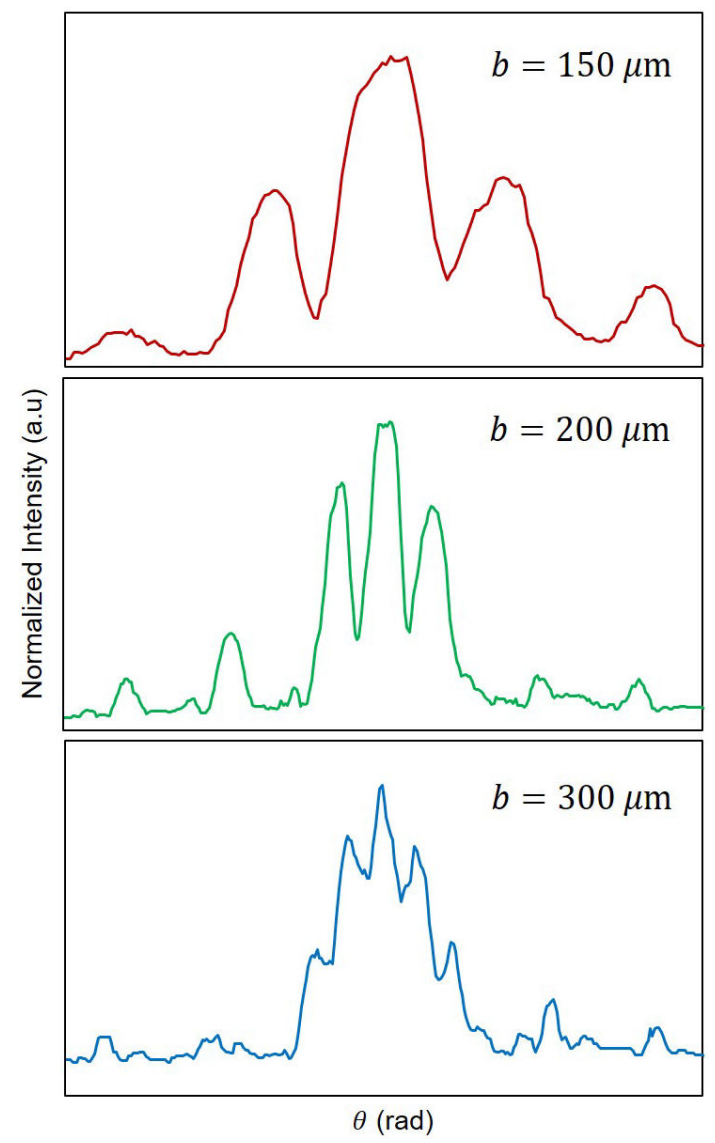

FIGURE 2. Experimental double slits diffraction pattern with various slit spacing. In the experiment, the distance between the slit and the screen $(L)$ is fixed, $2 \mathrm{~m}$. The slit width (d) used is $100 \mu \mathrm{m}$. The laser used is helium laser with $\lambda=6328 \AA$.

Using the same parameters with the experiment $(L=2$ $m, d=100 \mu \mathrm{m}$, and $\lambda=6328 \AA$ ), we plot the diffraction pattern at double slit according to Eq. (1) (see Fig. 3).

The experimental pattern is not as smooth as the theoretical pattern. It may be due to the limitation in the experiment itself or the limitation of the imaging technique. However, qualitatively the experimental pattern shows a similar pattern with the theoretical intensity profile for the diffraction at the double slits. As the slit spacing increases, the width of the central and nearby maxima becomes narrower. The location of the first and second maxima also shifts closer to $\theta=0$, as the slit spacing increases.

\subsection{Multiple slits experiment with a variation of slits number}

For multiple slits experiments, the number of slits $(N)$ is varied. The slit width $(d)$ and spacing $(b)$ are fixed to 50 and $75 \mu \mathrm{m}$, respectively. Figure 4 shows the diffraction pattern obtained from video analysis with Tracker. Whereas Fig. 5 shows the diffraction pattern got theoretically by plotting Eq. (1) with the same parameters used in the experiment ( $L=2 m, d=50 \mu \mathrm{m}, b=75 \mu \mathrm{m}$, and $\lambda=6328 \AA$ ).

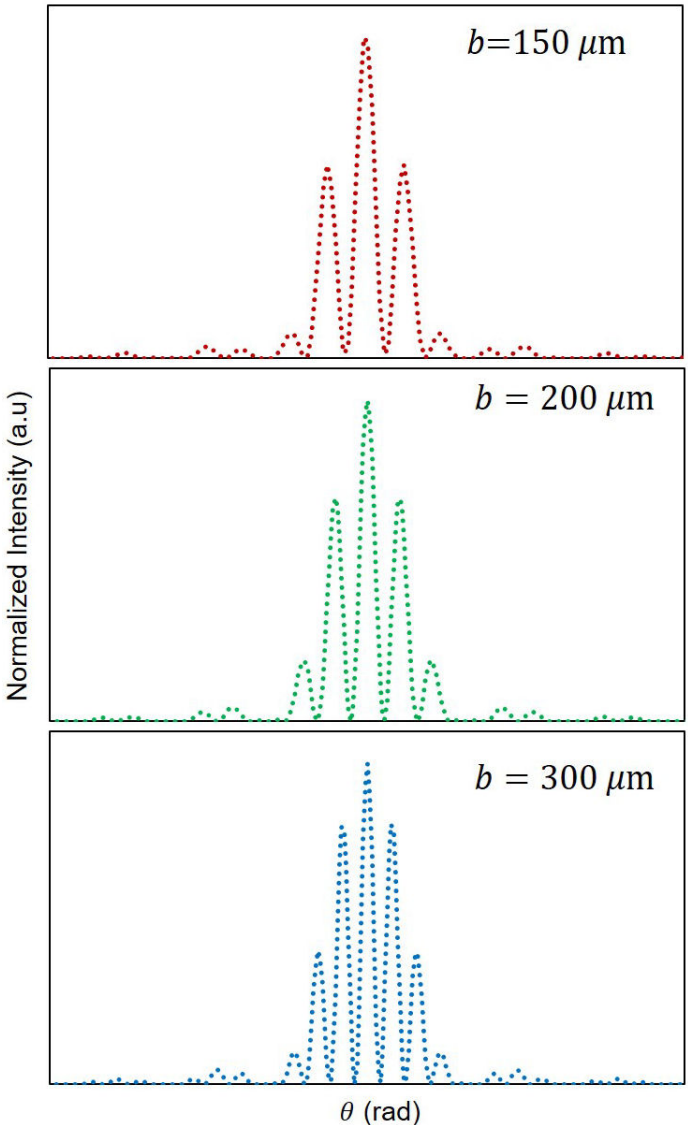

FIGURE 3. Theoretical double-slit diffraction pattern with various slit spacing. The used parameters are $L=2 \mathrm{~m}, d=100 \mu \mathrm{m}$, and $\lambda=6328 \AA$.

Similar to the double-slit experiment result, the experimental diffraction patterns at multiple slits are also not as smooth as the theoretical prediction. However, the same trend is observed. As the number of slits increases, the width of the maxima becomes narrower. For $N>2$, small peaks/small maxima arise between principal maxima.

\subsection{Diffraction grating experiment with a variation of grating constant}

The experiment is continued with the diffraction grating. In this experiment, the grating constant is varied. The used grating constants are 100, 200, and 300 lines/inch. The intensity of the Fraunhofer diffraction pattern is presented in Fig. 6. It can be seen that as the grating constant increases, the distance between principal maxima or fringe spacing become wider. By using the intensity profile in Fig. 6 and Eq. (3), we also can determine the wavelength of the laser. The calculated wavelength from the experiment is $6.160 \pm 0.037 \times 10^{-7} \mathrm{~m}$ (see Table I). This value is slightly below the reference value $\left(\lambda_{\text {ref }}=6.328 \times 10^{-7} \mathrm{~m}\right)$ that is given in the video explanation. The percentage of the discrepancy between the result of the laser wavelength found with Tracker, and the reference value is given in the video is about $2.6 \%$; which is quite small. 


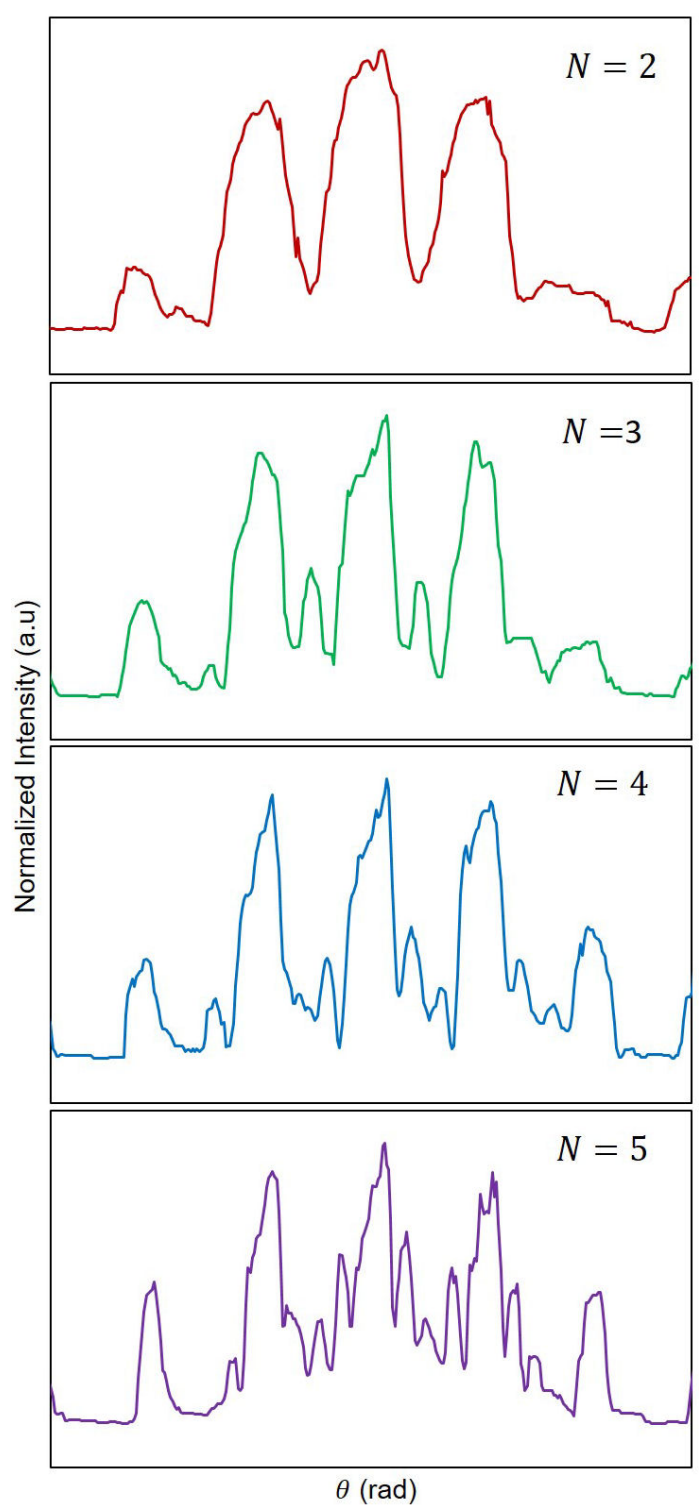

FIGURE 4. Experimental diffraction pattern with various slits number. In the experiment, the distance between the slit and the screen $(L)$ is fixed, $2 \mathrm{~m}$. The slit width $(d)$, and the slit spacing $(b)$ used are 50 and $75 \mu \mathrm{m}$, respectively. The laser used is helium laser with $\lambda=6328 \AA$.

\subsection{Implementation in learning process}

In distance learning, laboratory activity is hard to be conducted. Activity with video analysis and spreadsheets can be an alternative option. Through the activity, students can develop their science process skills and conceptual understanding in Fraunhofer diffraction. They also can improve mathematical and computational thinking skills.

In the teaching and learning process, the teacher can begin by presenting the Fraunhofer Diffraction video demonstration from MIT Open CourseWare. Meanwhile, students are asked to observe the Fraunhofer diffraction phenomena in the video. After the observation, the teacher can ask students to analyze the light spectra using the Tracker. However,

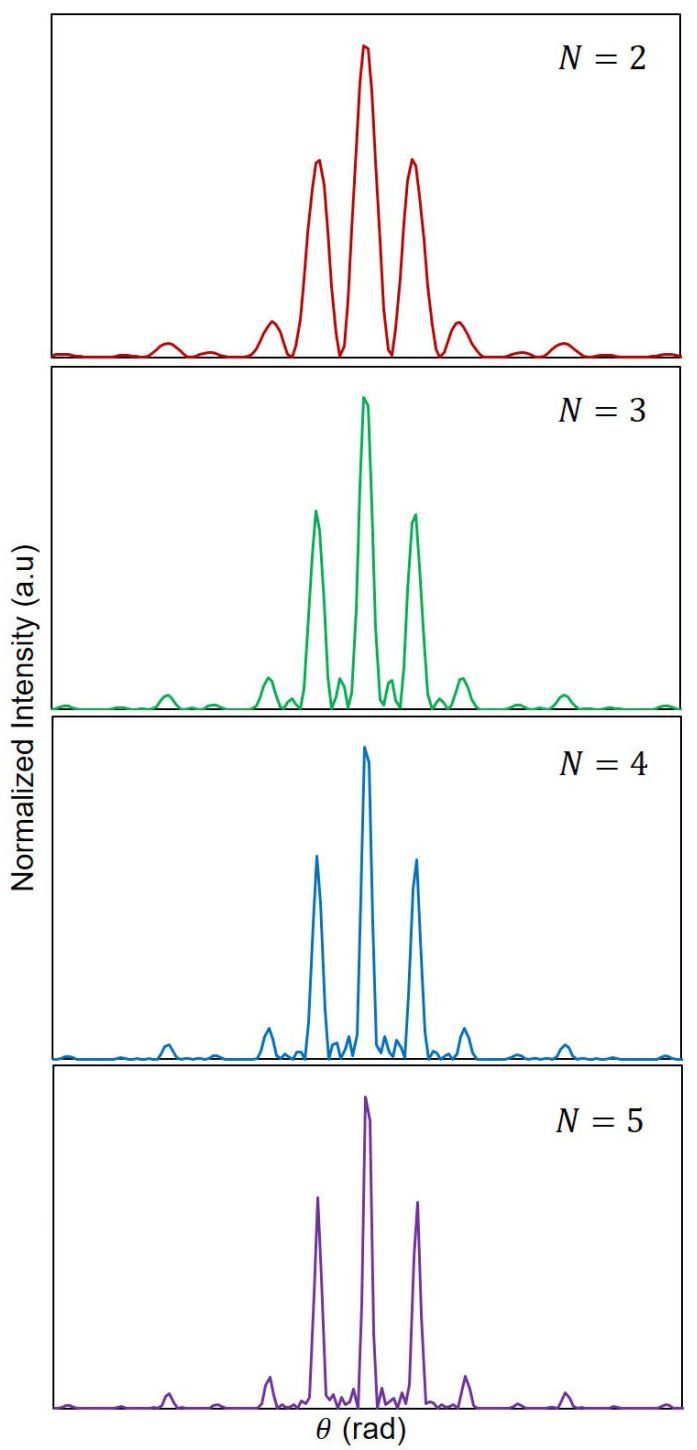

FIGURE 5. Theoretical diffraction pattern with various slits number. The used parameters are $L=2 \mathrm{~m}, d=50 \mu \mathrm{m}, b=75 \mu \mathrm{m}$, and $\lambda=6328 \AA$.

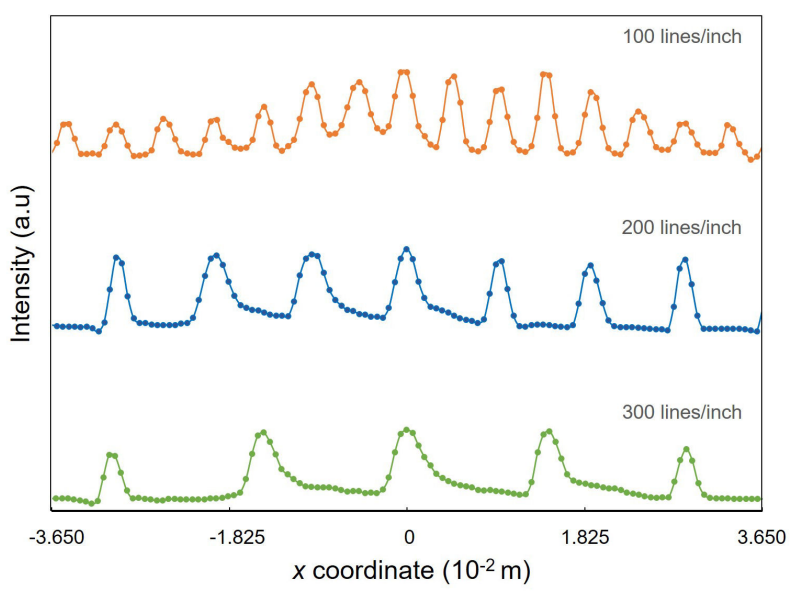

FIGURE 6. Intensity profile of Fraunhofer diffraction by grating with various grating constant. 
TABLE I. Wavelength Calculation

\begin{tabular}{ccccc}
\hline$L(\mathrm{~m})$ & $\Delta\left(\times 10^{-4} \mathrm{~m}\right)$ & $n$ & $S_{n}(\mathrm{~m})$ & $\lambda\left(\times 10^{-7} \mathrm{~m}\right)$ \\
\hline 2 & 2.540 & 1 & 0.0048 & 6.096 \\
2 & 1.270 & 1 & 0.0098 & 6.223 \\
2 & 0.847 & 1 & 0.0146 & 6.181 \\
\hline \multicolumn{5}{c}{$\bar{\lambda}=6.160 \pm 0.037 \times 10^{-7} \mathrm{~m}$} \\
\hline \multicolumn{5}{c}{ \%discrepancy $=\left(\left|\bar{\lambda}-\lambda_{\text {ref }}\right| / \lambda_{\text {ref }}\right) \times 100 \%=2.6 \%$}
\end{tabular}

teachers need to give an introduction to Tracker beforehand. After students get some findings, they should present their findings in the class forum. The activity can be continued with a discussion of Fraunhofer Diffraction through a mathematical approach. The teacher can encourage students to visualize the obtained mathematical equation with the spreadsheet. The discussion may enrich students with a theoretical explanation about their observation.

The video analysis activity can be carried out as a project or homework. This learning scenario will engage students more in 'virtual' physics experiments, and students may become more motivated in learning physics.

\section{Conclusion}

In this paper, we have presented the investigation of Fraunhofer diffraction with double and multiple slits through Tracker and spreadsheet program. Learning Fraunhofer diffraction using video Tracker and spreadsheet enables students to compare experimental and theoretical results. This activity has the potential to be used in distance learning to accommodate laboratory-related physics course.

\section{Acknowledgments}

The authors would like to thank Universitas Negeri Yogyakarta and Widya Mandala Catholic University for supporting this work.
1. S. Ezekiel, "Video Demonstrations in Lasers and Optics," (MIT OpenCourseWare, 2008).

https://ocw.mit.edu/resources/res-6-006 -video-demonstrations-in-lasers-and -optics-spring-2008/index.htm\#

2. D. Brown, "Tracker Video Analysis and Modeling Tool," (Physlets, 2020). https://physlets.org/tracker/

3. C. Sirisathitkul, P. Glawtanong, T. Eadkong, and Y. Sirisathitkul, "Digital Video Analysis of Falling Objects in Air and Liquid Using Tracker," Rev. Bras. Ensino Fis. 35 (2013) 1504. https://doi.org/10.1590/ S1806-11172013000100020

4. E. Yusuf, "Using Tracker to Engage Students' Learning and Research in Physics," Pertanika J. Sci. Technol. 24 (2016) 483491.

5. L. K. Wee, C. Chew, G. H. Goh, S. Tan, and T. L. Lee, "Using Tracker as a Pedagogical Tool for Understanding Projectile Motion," Phys. Educ. 47 (2012) 448-455. https : / / doi . org/10.1088/0031-9120/47/4/448

6. J. A. Bryan, "Investigating the Conservation of Mechanical Energy Using Video Analysis: Four Cases," Phys. Educ. 45 (2010) 50-57. https://doi.org/10.1088/0031-9120/45/ $1 / 005$

7. C. Berlic and V. Barna, "Arduino and Tracker Video-Didactic Tools for Study of The Kater Pendulum Physical Experiment," Rom. Reports Phys. 72 (2020) 901.

8. J. Poonyawatpornkul and P. Wattanakasiwich, "High-speed video analysis of damped harmonic motion," Phys. Educ. 782 (2013). https://doi.org/10.1088/0031-9120/ $48 / 6 / 782$

9. T. Eadkhong, R. Rajsadorn, P. Jannual, and S. Danworaphong "Rotational dynamics with Tracker," Eur. J. Phys. 33 (2012) 615-622. doi:10.1088/0143-0807/33/3/615
10. S. K. Ayop, "Analyzing Impulse Using iPhone and Tracker," Phys. Teach. 55 (2017) 480-481. https://doi.org/10. $1119 / 1.5008342$

11. M. Belloni, W. Christian, and D. Brown, "Teaching Astronomy Using Tracker," Phys. Teach. 51 (2013) 149-150. https : //doi.org/10.1119/1.4792008

12. M. Rodrigues and P. S. Carvalho, "Teaching optical phenomena with Tracker," Phys. Educ. 49 (2014) 671-677. https : //doi.org/10.1088/0031-9120/49/6/671

13. L. K. Wee, "Open Educational Resources from Performance Task using Video Analysis and Modeling-Tracker and K12 Science Education Framework," in Overseas Chinese Physicists and Astronomers, (2014). pp. 1-5.

14. P. Hockicko, L. Kriš ták, and N. Miroslav, "Development of students' conceptual thinking by means of video analysis and interactive simulations at technical universities," Eur. J. Eng. Educ. (2014) 37-41. https://doi.org/10.1080/ 03043797.2014 .941337

15. J. Benacka, "Projectile general motion in a vacuum and a spreadsheet simulation," Phys. Educ. 50 (2015) 58. https: //doi.org/10.1088/0031-9120/50/1/58

16. I. Singh, K. K. Khun, and B. Kaur, "Visualizing the trajectory of a charged particle in electric and magnetic fields using an Excel spreadsheet," Phys. Educ. 54 (2019) 015002. https://doi.org/10.1088/1361-6552/aae3fd

17. E. Hecht, Optics, 4th ed. (New York: Pearson Education, 2002).

18. S. Ezekiel, "Fraunhofer Diffraction-Two Slits," (MIT OpenCourseWare, 2008).

https://ocw.mit.edu/resources/res-6-006-v ideo-demonstrations-in-lasers-and-optics -spring-2008/demonstrations-in-physicaloptics/fraunhofer-diffraction-2014-two-slits/ 
19. S. Ezekiel, "Fraunhofer Diffraction - Multiple Slits," (MIT OpenCourseWare, 2008).

https://ocw.mit.edu/resources/res-6-006-v ideo-demonstrations-in-lasers-and-opticsspring-2008/demonstrations-in-physical-opt ics/fraunhofer-diffraction-2014-multiple-slits/ 